\title{
A natural variant in ANP32B impairs influenza virus replication in human cells
}

\author{
Ecco Staller†, Carol M. Sheppard, Laury Baillon, Rebecca Frise, Thomas P. Peacock, Vanessa Sancho-Shimizu and \\ Wendy S. Barclay*
}

\begin{abstract}
Viruses require host factors to support their replication, and genetic variation in such factors can affect susceptibility to infectious disease. Influenza virus replication in human cells relies on ANP32 proteins, which are involved in assembly of replicationcompetent dimeric influenza virus polymerase (FluPol) complexes. Here, we investigate naturally occurring single nucleotide variants (SNV) in the human Anp32A and Anp32B genes. We note that variant rs182096718 in Anp32B is found at a higher frequency than other variants in either gene. This SNV results in a D130A substitution in ANP32B, which is less able to support FluPol activity than wild-type ANP32B and binds FluPol with lower affinity. Interestingly, ANP32B-D130A exerts a dominant negative effect over wild-type ANP32B and interferes with the functionally redundant paralogue ANP32A. FluPol activity and virus replication are attenuated in CRISPR-edited cells expressing wild-type ANP32A and mutant ANP32B-D130A. We propose a model in which the D130A mutation impairs FluPol dimer formation, thus resulting in compromised replication. We suggest that both homozygous and heterozygous carriers of rs182096718 may have some genetic protection against influenza viruses.
\end{abstract}

\section{INTRODUCTION}

All viruses rely on host factors to support their replication. Genetic variation in such proteins can affect susceptibility to infectious disease [1-4]. Perhaps the best-known mutation conferring genetic resistance to viral infection is the $\Delta 32$ variant of the HIV-1 co-receptor C-C chemokine receptor type 5 (CCR5) [5]. Single nucleotide variants (SNV) that redutce susceptibility to virus infection include a P424A substitution in the filovirus endosomal fusion receptor Niemann-PickC1 (NPC1) [6,7], and a G428A mutation in fucosyltransferase 2 (FUT2) that renders homozygous carriers resistant to norovirus $[8,9]$. These variants affect viral entry - no SNVs resulting in defects in replication have thus far been described.

Genetic variation in the endosomal transmembrane protein IFITM3 has been associated with increased severity of infection with influenza virus. The single nucleotide polymorphism (SNP) rs12252 leads to a 21-amino acid N-terminal truncation through an alteration of the first splice acceptor site, and is perhaps the best known genetic variant affecting influenza disease severity [10-1]. Although the association has not always been clear $[13,14]$, a recent meta-analysis including 12 studies and $>16000$ subjects confirmed the link between the IFITM3 minority allele and influenza severity [15]. Another important polymorphism in the IFITM3 gene associated with severe influenza is located in the $5^{\prime}$ UTR and leads to transcriptional repression through increased CTCF binding to the promoter [16].

Some non-synonymous natural variants in the restriction factor MxA lost antiviral activity, and even exerted a dominant-negative effect on the antiviral activity of wild-type MxA, suggesting heterozygous carriers of these variants may be more susceptible to influenza infection [17]. Additional mutations associated with severe influenza (reviewed in $[1,4]$ ) have been described in the complement factor CD55/DAF [18], the pulmonary surfactant protein SFTPA2 [19], the protease TMPRSS2 [20], the endothelial transcriptional activator GATA2 [21], interferon regulators IRF7 and IRF9 [22-24], and the endosomal dsRNA receptor TLR3 [25-27]. 
Almost all the host genetic variation relevant to influenza that has been described is associated with exacerbating rather than alleviating influenza virus infection, a bias explained by the higher visibility of severe cases, and the opportunity to perform whole genome sequencing on patients with severe symptoms. Hence, besides a pair of polymorphisms in the non-coding region (NCR) of the Galectin-1 encoding gene LGALS1, which may confer some protection against H7N9 influenza A virus (IAV) in Chinese poultry workers [28, 29], no single nucleotide variants protective against influenza virus have thus far been described.

Influenza replication and transcription are carried out in the host cell nucleus by the virus-encoded heterotrimeric RNAdependent RNA polymerase (FluPol) (reviewed in $[30,31]$ ), which relies on host factors to support RNA synthesis [32]. Oligomerisation of trimeric FluPol molecules into dimers, trimers or even higher order complexes is now believed to be required for efficient replication [33-37].

Influenza viruses co-opt the host acidic nuclear phosphoproteins of 32 kilodaltons A (ANP32A) and ANP32B to support their replication [38-40]. ANP32A and ANP32B are functionally redundant in their support for influenza virus: in human cells lacking either but not both ANP32A (AKO) or ANP32B (BKO), virus proliferation is not impaired. In cells lacking both proteins (dKO), however, FluPol activity and virus replication are completely abrogated $[39,40]$. ANP32 proteins are expressed in many different human tissues, including fibroblasts and lung [41], and involved in a wide range of cellular processes, including apoptosis and transcriptional regulation (reviewed in [42]). All of the human paralogues - ANP32A, ANP32B and ANP32E - associate with histones [43-46], placing them at the chromatin within the nucleus.

ANP32A and ANP32B are 249 and 251 amino acids long, respectively, with two major domains: a structured $\mathrm{N}$-terminal leucine-rich repeat region (LRR) and an intrinsically disordered low-complexity acidic region (LCAR) [46-48]. Mutational analysis of ANP32 proteins of several species has shown that the identity of amino acid residues 129 and 130 dictates pro-viral function [39, 40, 49, 50]. ANP32 proteins that cannot be co-opted by influenza virus include human ANP32E, chicken ANP32B, and mouse ANP32A. What these orthologues have in common is divergence from the pro-viral dyad 129N-130D: human ANP32E has glutamate at position 129 (129E) rather than asparagine, chicken ANP32B has isoleucine at position 129 (129I) and asparagine at position $130(130 \mathrm{~N})$, and mouse ANP32A has alanine at position 130 $(130 \mathrm{~A})$, suggesting that these amino acids are important for pro-viral activity of ANP32 proteins.

Recent structural work depicts the ANP32A LRR domain as stabilising a replication-competent dimer of a viral promoter-bound 'replicating' FluPol heterotrimer $\left(\mathrm{FluPol}^{\mathrm{R}}\right)$ and an 'encapsidating' $\mathrm{FluPol}^{\mathrm{E}}$, which is proposed to receive the nascent viral RNA product synthesised by $\mathrm{FluPol}^{\mathrm{R}}$ [33]. More specifically, the N-terminal half of the LRR interacts with $\mathrm{FluPol}^{\mathrm{R}}$, while the C-terminal portion interacts with
FluPol ${ }^{\mathrm{E}}$, in effect bridging the dimer. The importance of residues 129 and 130 is explained by direct interaction with basic residues on the PB1 subunit of $\mathrm{FluPol}^{\mathrm{E}}$. Interaction of the LCAR domain of ANP32A with FluPol has also been postulated but remains unresolved [33, 47, 51].

Here we describe natural variation in the human $A n p 32 A$ and Anp $32 B$ genes, demonstrating that the naturally occurring SNV rs182096718 in Anp32B can confer protection from influenza virus replication by compromising the interaction between FluPol and the host factor. We demonstrate that rs182096718 exerts a dominant-negative effect over wildtype ANP32B and moreover interferes with the functionally redundant paralogue ANP32A through paralogue interference [52]. We propose a model explaining why influenza replication is impaired in cells expressing the mutant ANP32B protein.

\section{METHODS \\ Cell culture}

Human eHAP cells (Horizon Discovery) were cultured in Iscove's modified Dulbecco's medium (IMDM; Thermo Fisher) supplemented with $10 \%$ foetal bovine serum (FBS; Labtech), $1 \%$ nonessential amino acids (NEAA; Gibco), and $1 \%$ penicillin/streptomycin (Invitrogen). Human embryonic kidney (293T) cells (ATCC) and Madin-Darby canine kidney (MDCK) cells (ATCC) were maintained in Dulbecco's modified Eagle's medium (DMEM; Invitrogen) supplemented with $10 \%$ FBS, $1 \%$ NEAAs, and $1 \%$ penicillin/streptomycin. All cells were maintained at $37^{\circ} \mathrm{C}$ in a $5 \% \mathrm{CO}^{2}$ atmosphere.

\section{Plasmids and cloning}

Human FLAG-tagged pCAGGS-ANP32A and ANP32B expression plasmids have been described [39]. Mutant proteins were cloned from these plasmids by overlapping touchdown PCR, using primers CCAACCTGAATAACTA CCGCGAGAAC and GTTCTCGCGGTAGTTATTCA GGTTGG (ANP32A-D130N), GAATGACTACCAAGAG AACGTGTTC and GAACACGTTCTCTTGGTAGT CATTC (ANP32A-R132Q), GAGGCCCCTGATGCTG ACGCCGAGG and CCTCGGCGTCAGCATCAGGG GCCTC (ANP32A-S158A), GAGGCCCCTGATACTG ACGCCGAGGGC and GCCCTCGGCGTCAGTATCAG GGGCCTC (ANP32A-S158T), GTGACAAACGTGAATG ACTATCGG and CCGATAGTCATTCACGTTTGTCAC (ANP32B-L128V), CAAACCTGAATGCCTATCGG GAGAGC and GCTCTCCCGATAGGCATTCAGGTTTG (ANP32B-D130A), GACTATCGGCAGAGCGTGTTTAAG and CTTAAACACGCTCTGCCGATAGTC (ANP32BE133Q), GAGAGCGTGTTTAAGCACCTGCCACAGCTG and CAGCTGTGGCAGGTGCTTAAACACGCTCTC (ANP32B-L138H), GTTGCTGCCACAGTTTACTT ATCTCGA and TCGAGATAAGTAAACTGTGGCAGCAAC (ANP32B-L142F). Expression plasmids encoding H3N2 Vic/75 and pH1N1 RNP components PB1, PB2, PA and NP have been described [39], as have pCAGGS-ANP32 $\mathrm{A}_{\text {luc2 }}$ ' pCAGGS-luc1, pCAGGS-luc2, and H5N1 50-92 PB1 luc1C 
$[49,51]$. pPolI reporter plasmid containing firefly luciferase flanked by IAV-specific promoters, and pCAGGS-Renilla luciferase transfection / cellular transcription control have been previously described [39]. Expression plasmids pCAGGS-ANP32 $\mathrm{B}_{\text {luc } 2}, \quad$ ANP32A-D130N $\mathrm{N}_{\text {luc2 } 2}$, ANP32B-

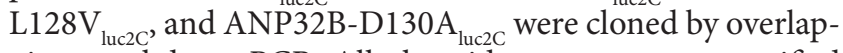
ping touchdown PCR. All plasmid constructs were verified by Sanger sequencing and analysed in Geneious prime 2019.

\section{CRISPR/Cas9 genome editing}

Guide RNA GCACTCTCTCGGTAGTCATTC was designed manually against the protospacer sequence in exon 4 of Anp32B to target DNA endonuclease SpCas9, expressed from Addgene plasmid \# 62988 (PX459), to the target nucleotide. The guide RNA itself was cloned into Addgene plasmid \# 80457 (pmCherry_gRNA). A custom-designed 88 base ssODN (single strand DNA) homology-directed repair template, harbouring the point mutation (cytosine in bold) and a silent PAM mutation (thymidine in italic script) - GAAAAGCC TGGACCTCTTTAACTGTGAGGTTACCAATCTGAATGC CTACCGAGAGAGTGTCTTCAAGCTCCTGCCCCAGCT TACCTAC - was obtained from Integrated DNA Technologies (IDT). Equal amounts of PX459 and pmCherry_gRNA (total $1 \mu \mathrm{g}$ ), and $1.0 \mu \mathrm{l} 10 \mu \mathrm{M}$ ssODN template, were transfected by electroporation into approximately $400000 \mathrm{eHAP}$ cells using the Neon transfection system (Invitrogen). Cells and DNA were mixed into a $10 \mu \mathrm{l}$ volume of suspension buffer, which was subjected to a single 1200 Volt pulse for a duration of $40 \mathrm{~ms}$. Cells were incubated at $37^{\circ} \mathrm{C}$ for $24 \mathrm{~h}$ in IMDM growth medium without antibiotics. A fluorescenceactivated cell sorter (FACS) Aria IIIU (BD Biosciences) with an $85 \mu \mathrm{m}$ nozzle was used to sort cells expressing mCherry (550-650 nm emission) into 96-well plates containing growth medium. Single cells were grown out into monoclonal populations over a period of 10 to 14 days. Total genomic DNA was extracted using the Purelink Genomic DNA Mini Kit (Invitrogen) and amplified by touchdown PCR to generate a 1561-base pair fragment of the edited locus (primers TACC TCTGCCCTCTCAATCTCT and ACGCACACAAACACAC ACTATT). PCR products were then incubated at $65^{\circ} \mathrm{C}$ for $30 \mathrm{~min}$ in the presence of BsmI restriction enzyme (NEB). The resulting DNA fragments were separated by $1.5 \%$ agarose gel electrophoresis. Potentially successfully edited clones were verified by Sanger sequencing (primers TAAAGACCGCTT GATACCCAGG and TGAGGCTGAGTGGGTAGTGG) and analysed in Geneious prime 2019.

\section{Minigenome reporter assays}

In order to measure influenza virus polymerase activity, pCAGGS expression plasmids encoding H3N2 Vic/75 or pH1N1 Eng/195 PB1 $(0.02 \mu \mathrm{g})$, PB2 $(0.02 \mu \mathrm{g})$, PA $(0.01 \mu \mathrm{g})$, and NP $(0.04 \mu \mathrm{g})$ were transfected into $\sim 100000 \mathrm{eHAP}$ cells using Lipofectamine 3000 (Thermo Fisher) at ratios of $2 \mu \mathrm{l}$ P3000 reagent and $3 \mu \mathrm{l}$ Lipofectamine 3000 reagent per microgram of plasmid DNA. As a reporter construct, we transfected $0.02 \mu \mathrm{g}$ pPolI-luc, which encodes a minigenome containing a firefly luciferase reporter flanked by influenza A virus promoter sequences. pCAGGS-Renilla luciferase $(0.02 \mu \mathrm{g})$ was co-transfected as a transfection and toxicity control. Amounts of co-transfected ANP32-FLAG constructs were $0.04 \mathrm{ug}$ (equal to pCAGGS-NP) unless otherwise specified in the Figure legends. Twenty-four hours after transfection, cells were lysed in $50 \mu$ l passive lysis buffer (Promega) for $30 \mathrm{~min}$ at room temperature with gentle shaking. Bioluminescence generated by firefly and Renilla luciferases was measured using the dual-luciferase system (Promega) on a FLUOstar Omega plate reader (BMG Labtech).

\section{Split luciferase complementation assay}

pCAGGS expression plasmids encoding H3N2 Vic/75 PB1luc1, PB2, PA, and the indicated ANP32-luc2 construct were transfected into $\sim 100000293 \mathrm{~T}$ cells at a ratio of 1:1:1:1 (15 ng per well). Control conditions contained pCAGGS-lucl and untagged PB1, or pCAGGS-luc2 and untagged ANP32A, respectively, with all other components remaining constant. Empty pCAGGS plasmid was used to ensure total transfected DNA was equal across conditions. Twenty-four hours after transfection, cells were lysed in $50 \mu$ Renilla lysis buffer (Promega) for $1 \mathrm{~h}$ at room temperature with gentle shaking (Gaussia and Renilla luciferase share the same substrate). Bioluminescence generated by Gaussia luciferase was measured using the Renilla luciferase kit (Promega) on a FLUOstar Omega plate reader (BMG Labtech). Normalized luminescence ratios (NLR) were calculated by dividing the signal from the potential interacting partners by the sum of the two controls, as described [51].

\section{Western blotting}

At least 250000 cells were lysed in buffer containing $50 \mathrm{mM}$ Tris-HCl (pH 7.8; Sigma-Aldrich), $100 \mathrm{mM} \mathrm{NaCl,} 50 \mathrm{mM}$ $\mathrm{KCl}$, and $0.5 \%$ Triton X-100 (Sigma-Aldrich), supplemented with a complete EDTA-free protease inhibitor cocktail tablet (Roche) and prepared in Laemmli $4 \times$ buffer (Bio-Rad) after protein concentration had been established by spectrophotometry (DeNovix DS-11 FX + spectrophotometer). Equal amounts of total protein (20-60 $\mu \mathrm{g}$ per lane) was resolved by SDS-PAGE using Mini Protean TGX precast gels $4-20 \%$ (Bio-Rad). Immunoblotting by semi-dry transfer (Bio-Rad Trans-Blot SD semidry transfer cell) onto nitrocellulose membranes (Amersham Protran Premium 0.2 $\mu \mathrm{m} \mathrm{NC;} \mathrm{GE}$ Healthcare) was carried out using the following primary antibodies: rabbit $\alpha$-vinculin (catalogue number ab129002, 1/2000; Abcam), rabbit a-ANP32A (catalogue number ab51013, 1/500; Abcam), rabbit a-ANP32B (10843-1-AP, 1/1000; Proteintech), rabbit $\alpha$-Gaussia luciferase (1/1000, Invitrogen) and mouse a-FLAG (catalogue number F1804, 1/500; Sigma-Aldrich). The following secondary antibodies were used: sheep $\alpha$-rabbit horseradish peroxidase (HRP) (catalogue number AP510P, 1/10000; Merck), goat $\alpha$-mouse HRP (STAR117P, 1/5000; AbD Serotec) and goat $\boldsymbol{\alpha}$-rabbit IgG IRdye800 (ab216773, 1/10000, Abcam). Protein bands were visualized by chemiluminescence using SuperSignal West 
Femto substrate (Thermofisher Scientific) on a Fusion-FX imaging system (Vilber Lourmat), or by fluorescence on a Licor Odyssey imager.

\section{Immunofluorescence microscopy}

Approximately 100000 eHAP cells were cultured on sterilised glass coverslips and transfected as per minigenome reporter assay protocol. Twenty-four hours after transfection, cells were fixed in $4 \%$ paraformaldehyde and permeabilized in $0.2 \%$ Triton X-100. FLAG-tagged ANP32 constructs were visualised with primary antibody mouse a-FLAG (F1804; 1/200; Sigma) for $2 \mathrm{~h}$ at $37^{\circ} \mathrm{C}$ in a humidified chamber. Cells were incubated with secondary antibody goat $\alpha$-mouse Alexa Fluor-568 (1/200; Life Technologies) for $1 \mathrm{~h}$ at $37^{\circ} \mathrm{C}$ in a humidified chamber, and counterstained with DAPI. Coverslips were mounted on glass slides using Vectashield mounting medium (H-1000-10; Vector Laboratories). Cells were imaged with a Zeiss Cell Observer widefield microscope with ZEN Blue software, using a Plan-Apochromat $\times 100$ 1.40-numerical aperture oil objective (Zeiss), an Orca-Flash 4.0 complementary metal-oxide semiconductor (CMOS) camera (frame, $2048 \times 2048$ pixels; Hamamatsu), giving a pixel size of $65 \mathrm{~nm}$, and a Colibri seven light source (Zeiss). Channels acquired and filters for excitation and emission were 4',6-diamidino-2-phenylindole (DAPI) (excitation [ex], 365/12 nm, emission [em] 447/60 nm), and TexasRed (ex 562/40 nm, em 624/40 nm). All images were analysed and prepared with Fiji software.

\section{Influenza virus infection}

A total of 500000 CRISPR/Cas9-modified monoclonal eHAP cells were infected with $\sim 2500$ plaque-forming units (PFU) H3N2 Vic/75 6:2 or pH1N1 Eng/195 6:2 virus diluted in $200 \mu \mathrm{l}$ serum-free IMDM for $1 \mathrm{~h}$ at $37^{\circ} \mathrm{C}$ (MOI 0.005) to allow virus to adsorb and enter the cells. The inoculum was removed and cells were incubated in room-temperature phosphate-buffered saline / $\mathrm{HCl}$ at $\mathrm{pH} 3.0$ for $3 \mathrm{~min}$ to inactivate residual virus. Cells were incubated at $37^{\circ} \mathrm{C}$ in serum-free cell culture medium (IMDM) supplemented with $1 \mu \mathrm{g} \mathrm{ml}^{-1}$ L-1-tosylamide-2-phenylethyl chloromethyl ketone (TPCK) trypsin (Worthington-Biochemical). Cell supernatants were harvested at indicated time points post-infection. Infectious titres were determined by plaque assay on MDCK cells. Virus infection assays were performed in triplicate on two separate occasions.

\section{Safety/biosecurity}

All work with infectious agents was conducted in biosafety level two facilities, approved by the Health and Safety Executive of the United Kingdom and in accordance with local rules, at Imperial College London, United Kingdom.

\section{Bioinformatics}

Human genomic information was obtained using the following publicly available databases: gnomAD (https:// gnomad.broadinstitute.org/); NCBI dbSNP (https://www. ncbi.nlm.nih.gov/snp/); ALSPAC (http://www.bristol.ac. uk/alspac/); TOPMed (https://www.nhlbiwgs.org/); 1000G (https://www.internationalgenome.org/); GO-ESP (https:// esp.gs.washington.edu/drupal/).

\section{RESULTS}

\section{A relatively common single nucleotide variant encodes ANP32B-D130A}

We searched public databases for missense SNVs in the human Anp32A and Anp32B genes that were predicted, on the basis of our previous work, to have functional impact on the pro-influenza virus activity of the proteins. We predominantly used the genome aggregation database gnomAD v2, which holds 125748 whole exomes and 15708 whole genomes from unrelated individuals sequenced in disease-specific or population genetic studies [53]. The gnomAD database identifies 54 missense SNVs in Anp32A and 82 in $A n p 32 B$ (observed/expected score of 0.39 vs 0.68 ), suggesting that Anp $32 \mathrm{~A}$ may be slightly more conserved. It is not currently possible to obtain specific phenotypic information about individual carriers of a particular SNV from gnomAD.

Four SNVs in Anp32A and five in $A n p 32 B$ were selected for investigation, taking into account changes in charge, bulk, polarity and putative solvent exposure of the encoded amino acid substitutions $[46,48]$, as well as the previously established importance of positions 129 and 130 for proinfluenza viral activity (Fig. 1a). All the variants in Anp $32 \mathrm{~A}$ are exceedingly rare (MAF $<0.001$ ), and no homozygous carriers have been identified. The serine mutants at position 158, S158A and S158T were of interest as this is a putative phosphorylation site [54]. The SNV responsible for a mutant ANP32A-D130N protein (rs771143708) is found in a single heterozygous individual out of 3854 in the Avon Longitudinal Study of Parents and Children (ALSPAC) cohort. This SNV is not described in the much larger gnomAD database, although it is cited in the NCBI database dbSNP. rs751239680 (ANP32A-R132Q) is found in a single heterozygous individual out of 10052 in the African cohort of the gnomAD database, rs772530468 (ANP32A-S158T) is present in a single South Asian male in 23070 in the gnomAD database, as well as two separate homozygous individuals in the Trans-omics for precision medicine (TOPMed) database [55]. Finally, a single African male in 5032 in the gnomAD database is a heterozygous carrier of rs772530468 (ANP32A-S158A) (Fig. 1b).

Most variants in $A n p 32 B$ were also low-frequency (MAF <0.0001): rs377406514 (ANP32B-L128V) is found in a single African female out of 10066 in the gnomAD database; rs771977254 (ANP32B-E133Q) occurs in two out of 4900 Ashkenazi Jewish females in the gnomAD database; rs761932651 (ANP32B-L138H) is found in one South Asian male in 23070 in the gnomAD database; rs770020996 (ANP32B-L142F) occurs in one female in 7544 and one male in 23072 from the South Asian cohort of the gnomAD database (Fig. 1c). 
(a)

\begin{tabular}{|l|l|l|l|l|l|l|l|}
\hline Gene & Variant & ID & \# alleles & MAF & \# homs & $\begin{array}{l}\text { Population } \\
\text { cohort }\end{array}$ & $\begin{array}{l}\text { Source } \\
\text { database }\end{array}$ \\
\hline Anp32A & D130N & 771143708 & $1 / 3,854$ & $2.59 \mathrm{e}-4$ & - & British & ALSPAC \\
\hline & R132Q & 751239680 & $1 / 121,042$ & $8.26 \mathrm{e}-6$ & - & African & ExAC \\
\hline & S158A & 772530468 & $1 / 250,554$ & $3.99 \mathrm{e}-6$ & - & African & gnomAD \\
\hline & S158T & 772530468 & $1 / 31,376$ & $3.19 \mathrm{e}-5$ & - & S Asian & gnomAD \\
\hline Anp32B & L128V & 377406514 & $1 / 251,030$ & $3.98 \mathrm{e}-6$ & - & African & gnomAD \\
\hline & D130A & 182096718 & $1233 / 282,628$ & $4.36 \mathrm{e}-3$ & 25 & Hispanic/Latino & gnomAD \\
\hline & E133Q & 771977254 & $2 / 251,352$ & $7.96 \mathrm{e}-6$ & - & $\begin{array}{l}\text { Ashkenazi } \\
\text { Jewish }\end{array}$ & gnomAD \\
\hline & L138H & 761932651 & $1 / 251,384$ & $3.98 \mathrm{e}-6$ & - & S Asian & gnomAD \\
\hline & L142F & 770020996 & $2 / 251,398$ & $7.96 \mathrm{e}-6$ & - & S Asian & gnomAD \\
\hline
\end{tabular}

(b)

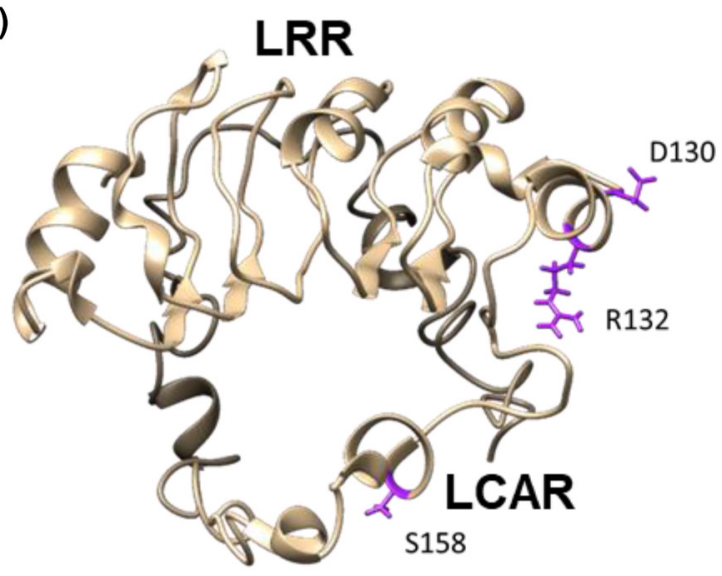

(c)

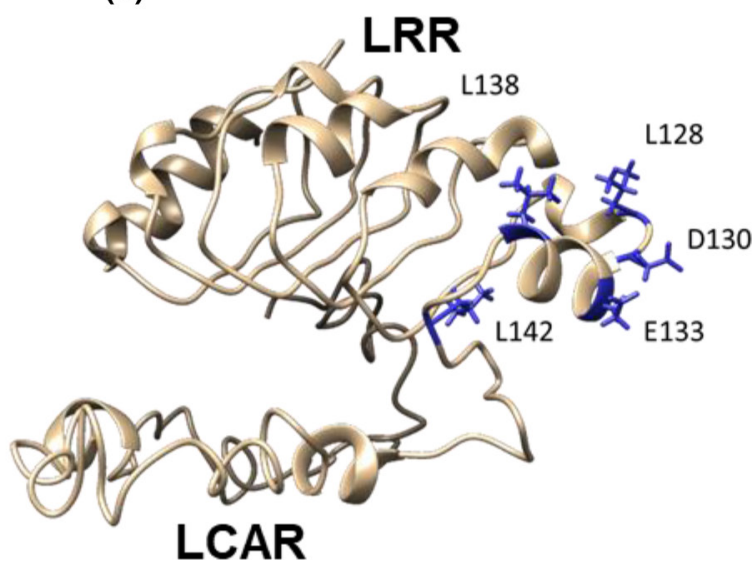

Fig. 1. Selected naturally occurring missense single nucleotide variants in Anp32A and Anp32B. (a) Amino acid substitutions (variant), NCBI dbSNP Reference IDs, number of minority alleles, global minor allele frequency (MAF), number of homozygous carriers (homs), gnomAD population cohort in which the variant is predominant, and the main source database for each variant. (b) Structural model of ANP32A highlighting in purple stick format amino acids affected by selected SNVs. (c) Structural model of ANP32B highlighting in blue stick format amino acids affected by selected SNVs. The models were generated using iTASSER structural prediction software based on deposited partial structures (GenBank 2RR6A (ANP32B), 2JQDA (ANP32A) and 2JEOA (ANP32A)), and created in UCSF Chimaera.

There was, however, an interesting exception. SNV rs182096718, encoding ANP32B-D130A, was relatively common in the Hispanic / Latino cohort of the gnomAD database, where in a total pool of 35420 individuals, 1209 heterozygous carriers were identified, as well as 25 homozygous carriers. The D130A substitution in ANP32B is thus present in $3.41 \%$ of the Latino cohort. The global MAF of this variant is 0.0044 , much higher than any other SNV in either Anp32A or Anp32B.

\section{Natural variants of ANP32 proteins affect support of FluPol activity}

We have previously described a method to measure the capacity of mutated ANP32 proteins to act as pro-viral factors for influenza polymerase activity. This is achieved by exogenous expression of the cloned mutants in human eHAP cells lacking ANP32A and ANP32B (dKO), in which influenza polymerase is unable to function in absence of complementing ANP32 $[39,56]$. Here we expressed each 


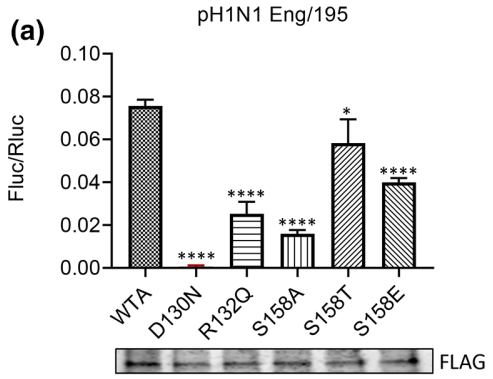

(b)

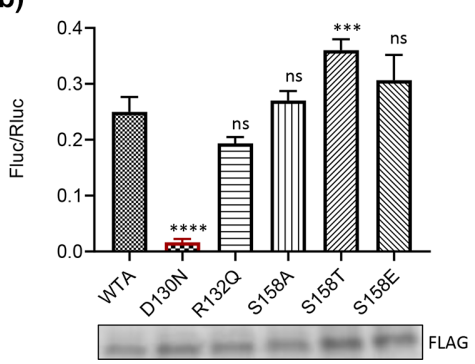

(c)

$$
\text { 仓) }
$$

(d)

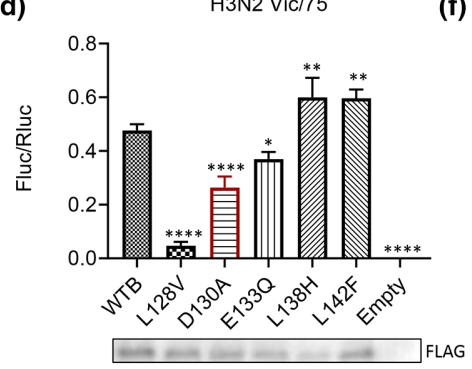

pH1N1 Eng/195

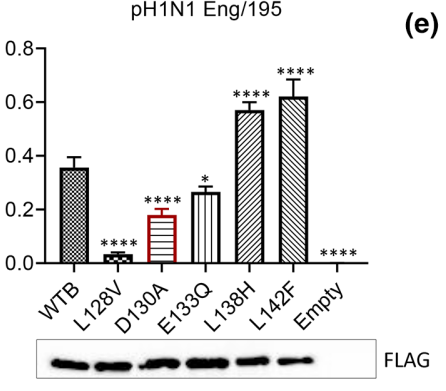

(f)

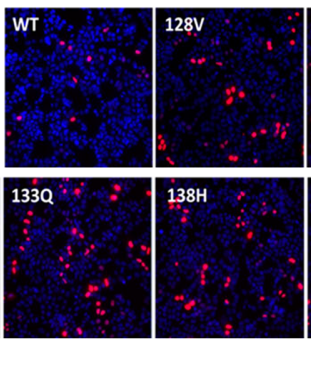

e)
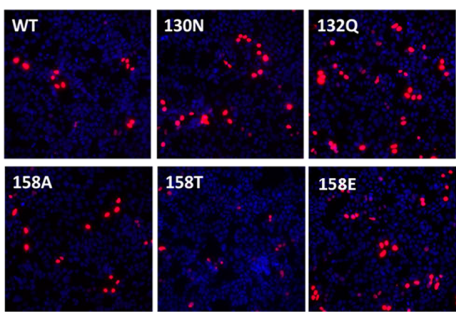

$130 \mathrm{~A}$

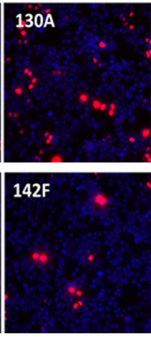

Fig. 2. ANP32A and $B$ mutant proteins show variable capacity to rescue IAV FluPol activity. (a-d) Minigenome reporter assays in eHAP dKO cells with co-transfected FLAG-tagged ANP32A (a, c) or ANP32B variants (b, d) with either pH1N1 Eng/195 (a, b) or H3N2 Vic/75 (c, d) RNP components PB1, PB2, PA and NP, pPoll-firefly luciferase minigenome reporter, and Renilla luciferase control in a 2:2:1:4:2:2 ratio. Data show mean (SD) of firefly activity normalized to Renilla and analysed by one-way ANOVA from the experiment shown ( $n=3$ independent triplicate experiments). ${ }^{*} P<0.05 ;{ }^{* *} P<0.01 ;{ }^{* * *} P<0.001 ;{ }^{* * * *} P<0.0001$. Accompanying Western blots show expression of the FLAG-tagged ANP32 constructs. (e-f) Immunofluorescence analysis showing nuclear localisation of FLAG-tagged ANP32A (e) or ANP32B (f) constructs, detected with anti-FLAG primary antibody and Alexa Fluor-568 anti-mouse conjugate and counterstained with DAPI.

natural ANP32A or ANP32B variant and tested its ability to support reconstituted polymerases from a 2009 pandemic H1N1 isolate (A/England/195/2009; hereafter 'Eng/195') or a seasonal $\mathrm{H} 3 \mathrm{~N} 2$ virus (A/Victoria/3/75; hereafter 'Vic/75') in minigenome reporter assays (Fig. 2).

Compared with wild-type ANP32A (WTA), ANP32AD130N did not support Eng/195 polymerase activity at all, while R132Q and S158A substitutions had significantly reduced capacity to support FluPol activity (Fig. 2a). An artificial mutant with the phosphomimic S158E also had reduced capacity to support Eng/195 polymerase. In contrast, ANP32A-S158T supported FluPol to an extent similar to wild-type ANP32A. Compared with wild-type ANP32B (WTB), ANP32B-D130A had a deleterious effect on the support for Eng/195 polymerase activity, as did the leucine to valine substitution at position 128 (ANP32B-L128V) (Fig. 2b). ANP32B-E133Q was also significantly less able to support Eng/195 FluPol activity. Substitutions of the leucines at positions 138 and 142 to histidine $(\mathrm{L} 138 \mathrm{H})$ and phenylalanine (L142F), respectively, did not compromise the ability of the mutant ANP32B proteins to support Eng/195 polymerase activity.

In general, the effects of natural variation in ANP32 proteins was similar for Vic/75 polymerase. ANP32A-D130N did not support Vic/75 polymerase activity, compared with WTA (Fig. 2c), but R132Q and S158A substitutions had a smaller effect on Vic/75 FluPol activity than on Eng/195 FluPol activity. The S158T substitution and the phosphomimic S158E were as capable of supporting Vic/75 FluPol activity as WTA. As seen with Eng/195 polymerase, the ANP32BL128V substitution was unable to rescue Vic/75 polymerase activity, but the D130A mutation had a detrimental effect and resulted in a reduction in Vic/75 polymerase activity (Fig. 2d). The observed differences in FluPol activity were not explained by differences in expression of the FLAG-tagged ANP32 constructs (Western blots accompanying Fig. 2a-d), nor by impaired nuclear localisation of the mutant proteins (Fig. 2e, f).

\section{ANP32 position 130 mutants show impaired binding to FluPol}

Using a split luciferase complementation assay previously developed in our laboratory [51] (Fig. 3a) we next assessed whether mutations at amino acid 130 affected interaction between ANP32 and FluPol. We and others have previously shown that murine ANP32A, which naturally harbours $130 \mathrm{~A}$, does not support influenza virus polymerase in minireplicon assays. Substituting 130A in human ANP32A greatly reduced FluPol activity, while introducing $130 \mathrm{D}$ in mouse ANP32A rescued its capacity to support FluPol activity [39, 40]. Indeed, ANP32B KO mice, but not ANP32A KO mice, showed reduced viral loads and mortality when infected with $\mathrm{H} 3 \mathrm{~N} 2$ or $\mathrm{H} 5 \mathrm{~N} 1$ influenza $\mathrm{A}$ virus [57]. Poor replication of H7N9 viruses in ANP32A 
(a)

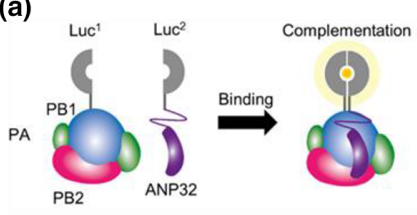

(b)

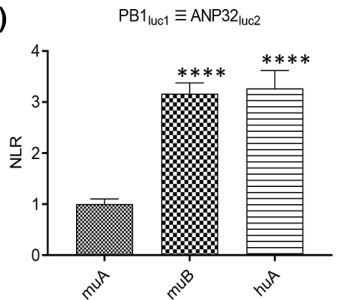

(c)

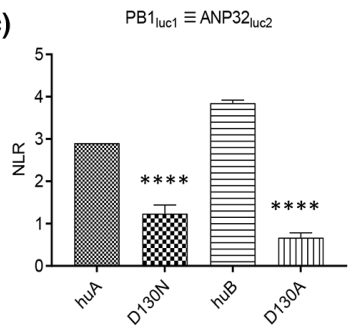

(d)

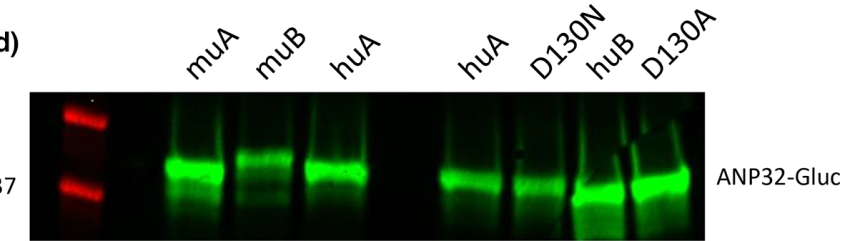

Fig. 3. ANP32 position 130 mutants show impaired binding to FluPol. (a) Schematic of split luciferase complementation assay (adapted from Mistry et al. 2020 [51]). (b) Interaction between mouse ANP32A (muA), mouse ANP32B (muB) or human ANP32A (huA) and Vic/75 FluPol. (c) Interaction between naturally occurring human ANP32 variants ANP32A-D130N (D130N) and ANP32B-D130A (D130A) and $\mathrm{Vic} / 75 \mathrm{FluPol}$, alongside their wild-type equivalents. Split luciferase complementation assays were carried out by transfecting equal amounts (15 ng) of pCAGGS expression plasmids encoding Vic/75 polymerase components PB1-luc1, PB2 and PA, as well as the indicated ANP32-luc2 construct into $293 \mathrm{~T}$ cells. At $24 \mathrm{~h}$ post-transfection cells were lysed and luminescence was measured. Total amount of plasmid (in ng) was kept constant by using empty pCAGGS. Normalised luminescence ratio (NLR) was obtained as described in Methods by dividing luminescence in the experimental condition (tagged PB1 +tagged ANP32) by the sum of the luminescence measured in the control conditions (i.e. background interaction of unbound luc1 with ANP32-luc2, and unbound luc2 with PB1-luc1, respectively). Data show mean (SD) of firefly activity normalized to Renilla and analysed by one-way ANOVA from the experiment shown ( $n=3$ independent triplicate experiments) ${ }^{* * *}, P<0.0001$. (d) Western blot showing expression of Gluc2-tagged ANP32 constructs.

knockout mice has also been described however [58]. Interestingly, interaction of murine ANP32A with FluPol was reduced $>2$-fold compared with mouse ANP32B, which does have pro-influenza virus activity, and with wild-type human ANP32A (Fig. 3b).

The naturally occurring human ANP32 variants ANP32AD130N and ANP32B-D130A also showed significantly reduced binding to FluPol (Fig. 3C). The luciferase signal indicating binding of ANP32A-D130N to FluPol was reduced $>2$-fold, relative to wild-type ANP32A, while the signal indicating FluPol interaction of ANP32B-D130A was greatly reduced compared with wild-type ANP32B. These differences in binding affinity were not due to differential expression of the ANP32-Gluc2 constructs (Fig. 3d).

\section{ANP32 position 130 mutants exert dominant- negative effects}

To investigate the functional consequence of the ANP32BD130A variant, we artificially recapitulated heterozygous and homozygous ANP32B-D130A variant genotypes by exogenous expression of the mutated proteins in CRISPRedited eHAP cells that lack ANP32B expression (BKO) [39] (Fig. 4a). Minigenome assays were performed with reconstituted pH1N1 Eng/195 polymerase complemented by transient transfection with increasing amounts of either wild-type ANP32B (homozygous wild-type; blue bars), ANP32B-D130A (homozygous mutant; red bars) or a 1:1 ratio of both (heterozygous mutant; purple bars). We found that adding increasing amounts of wild-type ANP32B, essentially mimicking the homozygous wildtype genotype (blue bars), had no effect on FluPol activity compared with FluPol supported by ANP32A alone (grey bar). This is presumably due to the presence of wild-type ANP32A in the BKO cells, since ANP32A and ANP32B serve redundant roles in supporting FluPol [39]. In contrast, polymerase activity decreased significantly when ANP32BD130A rather than wild-type ANP32B was co-transfected, and this effect was more dramatic the more variant was expressed (red bars). This suggests paralogue interference of ANP32B-D130A over wild-type ANP32A, which would otherwise support polymerase activity effectively on its own (grey bar). Co-transfecting increasing amounts of wildtype and mutant ANP32B in a 1:1 ratio, recapitulating the heterozygous mutant genotype (purple bars), also resulted in a significant drop in polymerase activity. This suggests that ANP32B-D130A also exerts a dominant-negative effect over wild-type ANP32B. These observations were unrelated to expression levels of the FLAG-tagged ANP32 constructs (accompanying Western blots). Statistical significance of the differences in Eng/195 polymerase activity between conditions is shown in the accompanying Table.

We next investigated whether similar dominant-negative effects were exerted by the rare ANP32A-D130N variant (Fig. 4b). In eHAP cells lacking ANP32A expression 
(a)

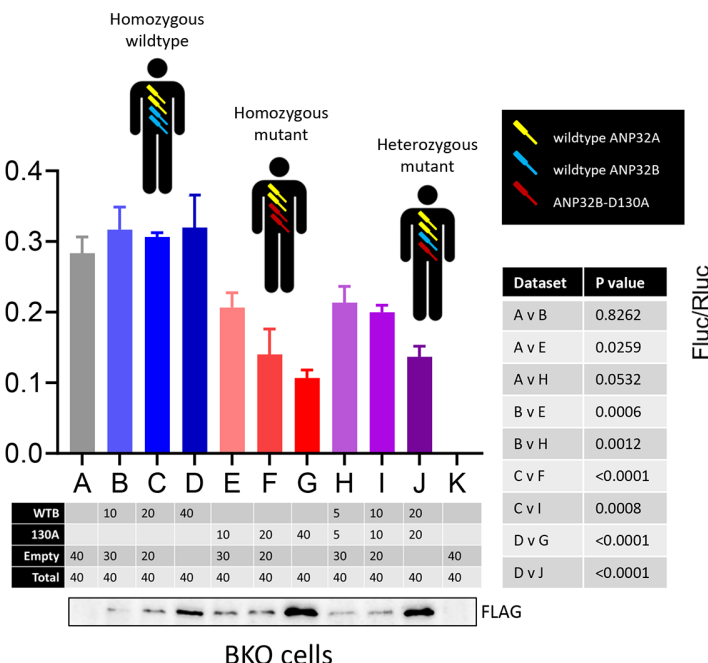

(b)

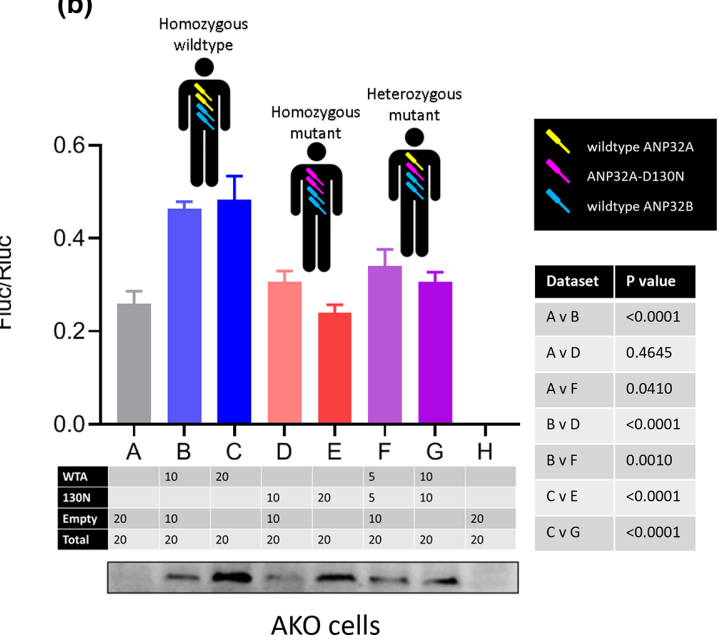

Fig. 4. ANP32B-D130A exerts dominant-negative effects on proviral function of wild-type ANP32B and ANP32A. (a) Minigenome reporter assay in eHAP cells lacking ANP32B expression (BKO) with 2009 pH1N1 Eng/195 RNP components PB1, PB2, PA and NP, pPoll-firefly luciferase minigenome reporter, and Renilla luciferase control, in a 2:2:1:4:2:2 transfection ratio with $20 \mathrm{ng}$ pCAGGS-PB1 and $10 \mathrm{ng}$ pCAGGS-PA per well $\left(\sim 1.0 \times 10^{5}\right.$ cells). Complementation by co-transfection of indicated combinations of wild-type ANP32B, ANP32BD130A, or empty pCAGGS plasmid (in ng) is shown in the Table. Total amounts of transfected plasmid DNA were kept constant across conditions by supplementing with empty pCAGGS plasmid. Accompanying Western blot shows expression of the FLAG-tagged ANP32 constructs. Cartoon figures show the wild-type and mutant genotypes found in carriers of SNV rs182096718. All three genotypes have two alleles of wild-type Anp32A (yellow). Homozygous ANP32B wild-type individuals carry two wild-type Anp32B alleles (blue); homozygous mutant carriers have two alleles encoding ANP32B-D130A (red), and finally heterozygous mutant carriers have one wild-type and one variant Anp32B allele. (b) Minigenome reporter assay in eHAP cells lacking ANP32A expression (AKO) with 2009 pH1N1 Eng/195 RNP components PB1, PB2, PA and NP, pPoll-firefly luciferase minigenome reporter, and Renilla luciferase control, in a 2:2:1:4:2:2 transfection ratio. Co-transfected wild-type ANP32A, ANP32A-D130N and empty pCAGGS plasmid are indicated in the Table, as in (a). Accompanying Western blot shows expression of the FLAG-tagged ANP32 constructs. Cartoon figures show the wild-type and mutant genotypes found in carriers of SNV rs771143708. All three genotypes have two alleles of wild-type Anp32B (blue). Homozygous ANP32A wild-type individuals carry two wild-type Anp32A alleles (yellow); hypothetical homozygous mutant carriers have two alleles encoding ANP32A-D130N (pink), and finally heterozygous mutant carriers have one wild-type and one variant Anp32A allele. Data show mean (SD) of firefly activity normalized to Renilla and analysed by one-way ANOVA from the experiment shown ( $n=3$ independent triplicate experiments).

(AKO), pH1N1 Eng/195 polymerase activity increased significantly when wild-type ANP32A was provided by transient co-transfection (blue bars), compared with FluPol activity supported by ANP32B alone (grey bar). In contrast, decreased polymerase activity was evident when either ANP32A-D130N alone (red bars) or wild-type and mutant ANP32A in a 1:1 ratio (purple bars) were co-transfected, albeit not in comparison with the ANP32B-only condition (grey bar). This suggests that, like ANP32B-D130A, ANP32A-D130N is dominant-negative over wild-type ANP32A, although there is no evidence that it also exerts paralogue interference over wild-type ANP32B. Together these data show that different substitutions of the canonical aspartate at position 130 of both pro-influenza viral human ANP32 proteins can have comparable phenotypic effects and may impact influenza proviral activity even in the heterozygous condition.

\section{IAV polymerase activity and replication are attenuated in ANP32B-D130A mutant cells}

To further probe the potential significance of the relatively common ANP32B-D130A variant, we generated a cell line with the wild-type Anp32A / mutant Anp32B (D130A) genotype by CRISPR/Cas9 genome editing, using human codon-optimised SpCas9 and a single-stranded DNA (ssODN) homology-directed repair template. We also generated a cell line lacking the entire pro-viral 128-130 loop $(\Delta 128-130)$ (Fig. 5a). We selected an unsuccessfully edited clone to serve as a negative control, and carried out Western blotting analysis to ensure endogenous wild-type ANP32A and wild-type or mutant ANP32B were expressed in control and mutant cell lines (Fig. 5b). Using minigenome reporter assays, we found that reconstituted IAV polymerases from Eng/195 (Fig. 5c) or Vic/75 (Fig. 5d) were significantly less active in edited ANP32BD130A and $\Delta 128-130$ cells, compared with control cells. As previously described [39], polymerase activity was not attenuated in $\mathrm{BKO}$ cells, again suggesting paralogue interference of mutant ANP32B over wild-type ANP32A. In other words, IAV FluPol activity was higher in cells completely lacking ANP32B expression than in cells expressing the D130A mutant. As described previously [39], no polymerase activity was seen in cells lacking both ANP32A and ANP32B (dKO), nor in the negative control 
(a)

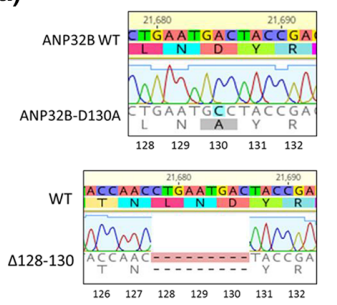

(d)

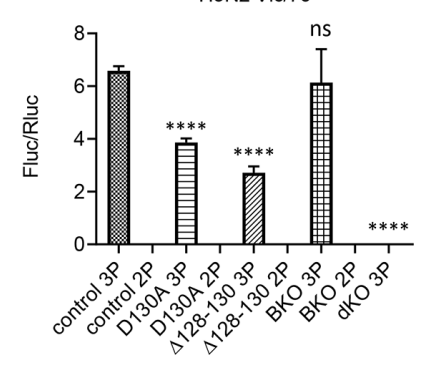

(b)

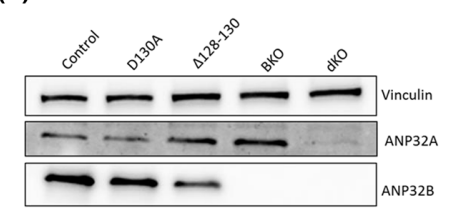

(e)

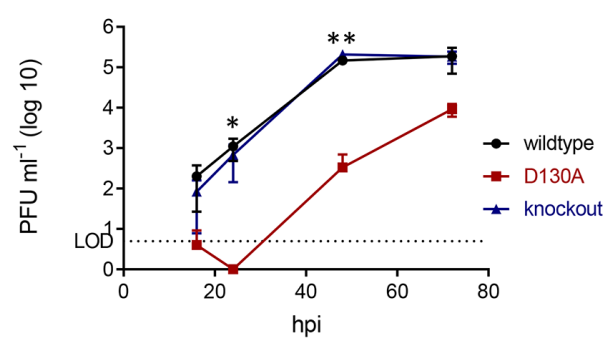

(c)

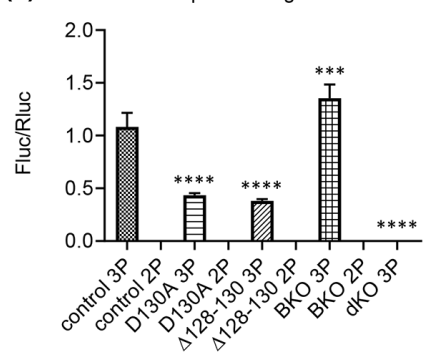

(f)

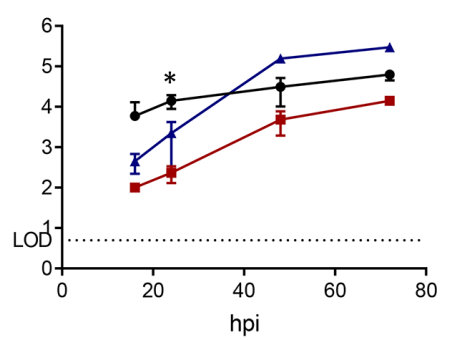

Fig. 5. FluPol activity and virus replication are attenuated in ANP32B-D130A mutant cells. (a) Genotypic profiles of CRISPR-edited monoclonal eHAP cell lines expressing ANP32B-D130A (top) or ANP32B lacking the pro-influenza virus 128-130 loop (D128-130). (b) Western blotting analysis showing expression of endogenous ANP32A and ANP32B, alongside vinculin loading control, in CRISPR-edited monoclonal control, ANP32B-D130A and ANP32B- $128-130$ cell lines, as well as the previously established ANP32B knockout (BKO) and ANP32A and B double knockout (dKO) cell lines. (c-d) Minigenome reporter assays in CRISPR-edited cell lines with pH1N1 Eng/195 (c) or H3N2 Vic/75 (d) RNP components PB1, PB2, PA and NP, pPoll-firefly luciferase minigenome reporter, and Renilla luciferase control, in a 2:2:1:4:2:2 ratio. Data show mean (SD) of firefly activity normalized to Renilla analysed by one-way ANOVA from the experiment shown ( $n=3$ independent triplicate experiments). The 3P, FluPol subunits PB1, PB2 and PA; 2P, negative control lacking FluPol subunit PB2. (e-f) CRISPR-edited control (wild-type; black), mutant ANP32B-D130A (D130A; red), and ANP32B knockout (blue) monoclonal cell lines were infected with either England/195 or Victoria/75 6:2 reassortant viruses with PR8 hemagglutinin (HA) and neuraminidase (NA) external genes at $\mathrm{MOI}=0.005$, and incubated at $37^{\circ} \mathrm{C}$ in the presence of $1 \mu \mathrm{g} \mathrm{ml} \mathrm{l}^{-1}$ trypsin to allow multicycle replication. Supernatants were harvested at the indicated times post-infection and pfu/ml established by plaque assay on MDCK cells. LOD (dotted line) denotes the limit of detection in the plaque assays. Shown are representative data from one of two independent infection experiments carried out in triplicate. P values were calculated by one-way ANOVA with Dunnet's multiple comparison test.

conditions where the polymerase subunit PB2 was lacking (2P).

Next we infected mutant (ANP32B-D130A), ANP32B knockout, or control cells at low MOI (0.005) with 6:2 recombinant influenza $A$ viruses containing the internal genes from $\mathrm{pH} 1 \mathrm{~N} 1 \mathrm{Eng} / 195$ and H3N2 Vic/75, with the neuraminidase (NA) and haemagglutinin (HA) external genes of the laboratory-adapted H1N1 PR/8 virus. Recombinant rather than wild-type viruses were used to enable us to carry out the work at biosafety level two. Eng/195 6:2 virus replication was severely attenuated in mutant D130A cells, in comparison with either control or BKO cells (Fig. 5e). Plaques were below the level of detection (LOD) 16 and $24 \mathrm{~h}$ post-infection and still three logs lower than control cells $48 \mathrm{~h}$ after infection $(P=0.0012)$. Vic/75 $6: 2$ infectious virus production in mutant cells was also lower than in control cells (Fig. 5f) but less affected than Eng/195. Both viruses replicated to higher titres in cells that completely lacked ANP32B (BKO) than in cells expressing the ANP32B-D130A mutant, suggesting paralogue interference of ANP32B-D130A over wild-type ANP32A also in an infectious virus context.

\section{DISCUSSION}

Here, we used publicly available databases to perform a biassed screen for naturally occurring single nucleotide variants in human $A n p 32 A$ and $A n p 32 B$, under the hypothesis that some mutations may affect susceptibility to influenza virus infection. One of the mutations, which translates to an aberrant ANP32B-D130A protein, was highly enriched in carriers of Latino descent. Although most carriers of this SNV (>1200) are heterozygous for the mutation, 25 homozygotes have been reported so far in the gnomAD database. Almost all carriers occur in the Hispanic/Latino subpopulation. We generated this naturally occurring homozygous genotype by CRISPR/Cas9 genome editing in low-ploidy human eHAP cells, and found that IAV FluPol activity and virus replication were significantly attenuated in the mutant cell line, compared to wild-type control cells or cells lacking ANP32B. This effect was most pronounced with the 2009 pandemic H1N1 strain Eng/195, which may be due to subtle differences in the interaction surfaces of the FluPol trimer. Importantly, we demonstrate that the mutant ANP32B-D130A protein exerts a dominant-negative effect over wild-type ANP32B, as well as interference over the functionally redundant paralogue 


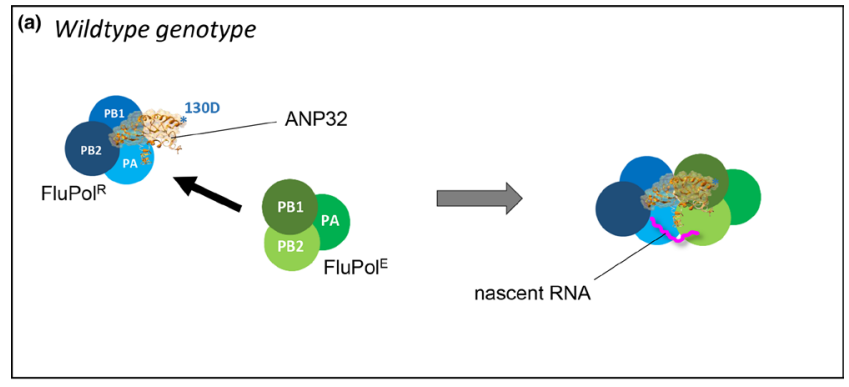

(b) Mutant genotype

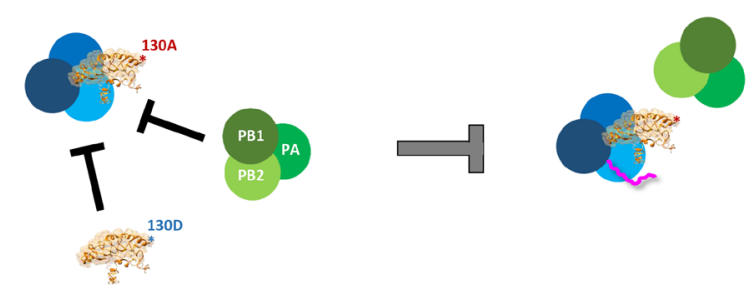

Fig. 6. Mutation at ANP32 position 130 results in impaired formation of replication-competent FluPol dimer. Cartoon explaining why FluPol activity is impaired in cells harbouring ANP32B-D130A. (a) Wild-type (130D) ANP32B (or ANP32A) acts by binding to a viral promoter-bound replicating FluPol (FluPol ${ }^{R}$, in blue) via the $\mathrm{N}$-terminal region of the LRR domain, while the C-terminal LRR, harbouring the surface-exposed acidic residue 130D, is used to recruit the encapsidating FluPol (FluPolE, in green), via direct interaction with basic residues on the PB1 subunit of FluPolE. In the context of a properly formed FluPol ${ }^{\mathrm{R}}$-ANP32-FluPol ${ }^{\mathrm{E}}$ complex the nascent RNA product replicated by FluPol $^{R}$ (in pink) transfers to FluPolE and replication proceeds efficiently. (b) The mutant ANP32B-D130A can still bind efficiently - and therefore competitively - to FluPol ${ }^{R}$, as the N-terminal portion of the LRR is unaffected, but because there is now an alanine in place of the acidic aspartate at position 130, recruitment of FluPolE is impaired. Furthermore, binding of ANP32B-D130A to FluPol ${ }^{R}$ prevents recruitment of wild-type ANP32A or $A N P 32 B$, explaining the paralogue interference and dominantnegative effects, respectively. Thus, in the presence of ANP32B-D130A, the replication-competent FluPol ${ }^{R}$-ANP32-FluPolE complex is not formed as efficiently, and therefore replication is compromised.

ANP32A. This suggests that despite the redundancy in proinfluenza viral function of ANP32A and B, even in heterozygotes there may be some consequence of this genetic variation to outcome of virus infection. We provide the first example of a single nucleotide variant in the coding region of a human gene that might confer some protection against influenza virus infection.

Although we and others have previously reported redundancy in reconstituted systems for ANP32A and B in their support of FluPol activity $[39,40]$, the data presented here imply that deleterious mutations in one ANP32 protein might exert dominant-negative effects on the pro-viral activity of the other, a phenomenon known as paralogue interference. Poor interaction with FluPol of one paralogue might be expected to make no difference when both paralogues are present in the cell, but clearly this does seem to be the case.
Recent structural data suggest that ANP32 proteins assist influenza polymerase by stabilizing a dimer between the replicating FluPol trimer, $\mathrm{FluPol}^{\mathrm{R}}$, and a second trimer that is recruited as an encapsidating complex, $\mathrm{FluPol}^{\mathrm{E}}$ [33]. To explain the dominant-negative effects we observe here, as well as the compromised replicative activity, we propose a model in which the mutant ANP32B-D130A binds competitively to FluPol ${ }^{\mathrm{R}}$, via the unaltered $\mathrm{N}$-terminal portion of the LRR domain, but is unable to recruit $\mathrm{FluPol}^{\mathrm{E}}$ due to the lack of acidity at position 130 (Fig. 6). Consequently, nascent viral RNA generated by $\mathrm{FluPol}^{\mathrm{R}}$ is not transferred effectively to $\mathrm{FluPol}^{\mathrm{E}}$, and replication is compromised. Furthermore, recruitment of ANP32B-D130A to $\mathrm{FluPol}^{\mathrm{R}}$ occupies the binding site for proviral wild-type ANP32A or ANP32B proteins, explaining the paralogue interference and dominant-negative effects we observe.

It is important to bear in mind that the outcome of genetic variation will depend on gene dosing in relevant tissues. The genotype-tissue expression (GTEx) portal uses whole genome sequencing and RNA-Seq data to estimate gene expression in 54 non-diseased human tissue types from nearly 1000 individuals [41]. ANP32A and ANP32B are both clearly detectable in healthy lung tissue, at a median transcripts per kilobase million (TPM) of 42 and 201, respectively. There are thus 4.8 -fold more ANP32B transcripts than ANP32A transcripts in the lung, suggesting that mutations that impair pro-viral function of ANP32B, such as ANP32B-D130A, might have significant effects on virus replication in the target tissue. Moreover, ANP32B is more stable than ANP32A, judging from half-life measurements of both paralogues [59].

A caveat of the work presented here is the use of human eHAP cells where (primary) respiratory epithelium would have been preferable. We selected eHAP cells for their low ploidy, which renders CRISPR/Cas9 genome editing more straightforward, in the knowledge that more commonly used laboratory cell lines like A549 or HEK-293 derivatives are polyploid [60-62]. We and others have, however, shown previously that eHAP cells are a suitable model for influenza virus infection $[33,39,49-51]$. A drawback of using a low-ploidy cell line is that heterozygous genotypes cannot easily be generated. Thus far we have been unable to trace carriers of the variants but future work using diploid respiratory epithelial cells that reflect the heterozygous genotype could indicate whether true heterozygotes, like the reconstituted cells we used here, also display reduced influenza susceptibility.

In conclusion, we have provided the first example of a single nucleotide variant in the coding region of a human gene that may offer carriers some protection against influenza virus. This work has the potential to inform future intervention.

\section{Funding information}

E.S. was supported by an Imperial College President's Scholarship. L.B. was supported by Wellcome Trust grant 209213/Z/17/Z. R.F. was supported by Wellcome Trust grant 200187/Z/15/Z. T.P.P. was supported by BBSRC grant BB/R013071/1. V.S-S. was supported by UKRI Future Leaders Fellowship MR/S032304/1. C.M.S. and W.S.B. were funded by Wellcome Trust grant 205100 and BBSRC grant BB/K002456/1. 


\section{Acknowledgements}

The authors wish to thank David Gaboriau for help with microscopy, and the St. Mary's NHLI FACS core facility for support, instrumentation, and help with single cell sorting. The Facility for Imaging by Light Microscopy (FILM) at Imperial College London is partially supported by funding from the Wellcome Trust (grant 104931/Z/14/Z) and BBSRC (grant BB/L015129/1)

\section{Conflicts of interest}

The authors declare that there are no conflicts of interest.

\section{References}

1. Ciancanelli MJ, Abel L, Zhang SY, Casanova JL. Host genetics of severe influenza: from mouse Mx1 to human IRF7. Curr Opin Immunol 2016;38:109-120.

2. Karlsson EK, Kwiatkowski DP, Sabeti PC. Natural selection and infectious disease in human populations. Nat Rev Genet 2014;15:379-393.

3. Pittman KJ, Glover LC, Wang L, Ko DC. The legacy of past pandemics: common human mutations that protect against infectious disease. PLoS Pathog 2016;12.

4. Zhang Q. Human genetics of life-threatening influenza pneumonitis. Hum Genet 2020;139:941-948.

5. Carrington M, Dean M, Martin MP, O'Brien SJ. Genetics of HIV-1 infection: Chemokine receptor CCR5 polymorphism and its consequences. Hum Mol Genet 1999:8:1939-1945.

6. Carette JE, Raaben M, Wong AC, Herbert AS, Obernosterer G, et al. Ebola virus entry requires the cholesterol transporter NiemannPick C1. Nature 2011;477:340-343.

7. Kondoh T, Letko M, Munster VJ, Manzoor R, Maruyama J, et al. Single-nucleotide polymorphisms in human NPC1 influence filovirus entry into cells. J Infect Dis 2018:218:S397-s402.

8. Lindesmith L, Moe C, Marionneau S, Ruvoen N, Jiang $\mathrm{X}$, et al. Human susceptibility and resistance to Norwalk virus infection. Nat Med 2003:9:548-553.

9. Thorven M, Grahn A, Hedlund K-O, Johansson H, Wahlfrid C, et al. A homozygous nonsense mutation $(428 \mathrm{G} \rightarrow \mathrm{A})$ in the human secretor (FUT2) gene provides resistance to symptomatic norovirus (GGII) infections. J Virol 2005;79:15351-15355.

10. Everitt AR, Clare S, Pertel T, John SP, Wash RS, et al. IFITM3 restricts the morbidity and mortality associated with influenza. Nature 2012;484:519-523.

11. Lee N, Cao B, Ke C, Lu H, Hu Y, et al. IFITM3, TLR3, and CD55 gene SNPs and cumulative genetic risks for severe outcomes in Chinese patients with H7N9/H1N1pdm09 influenza. J Infect Dis 2017:216:97-104.

12. Zhang Y-H, Zhao Y, Li N, Peng Y-C, Giannoulatou E, et al. Interferoninduced transmembrane protein-3 genetic variant rs12252-C is associated with severe influenza in Chinese individuals. Nat Commun 2013:4:1418.

13. Mills TC, Rautanen A, Elliott KS, Parks T, Naranbhai V, et al. IFITM3 and susceptibility to respiratory viral infections in the community. $J$ Infect Dis 2014;209:1028-1031.

14. Randolph AG, Yip WK, Allen EK, Rosenberger CM, Agan AA, et al. Evaluation of IFITM3 rs12252 association with severe pediatric influenza infection. J Infect Dis 2017;216:14-21.

15. Prabhu SS, Chakraborty TT, Kumar N, Banerjee I. Association between IFITM3 rs12252 polymorphism and influenza susceptibility and severity: A meta-analysis. Gene 2018;674:70-79.

16. Allen EK, Randolph AG, Bhangale $T$, Dogra $P$, Ohlson $M$, et al. SNP-mediated disruption of CTCF binding at the IFITM3 promoter is associated with risk of severe influenza in humans. Nat Med 2017;23:975-983

17. Graf L, Dick A, Sendker F, Barth E, Marz M, et al. Effects of allelic variations in the human myxovirus resistance protein $A$ on its antiviral activity. J Biol Chem 2018;293:3056-3072.

18. Chatzopoulou F, Gioula G, Kioumis I, Chatzidimitriou D, Exindari M. Identification of complement-related host genetic risk factors associated with influenza A(H1N1)pdm09 outcome: challenges ahead. Med Microbiol Immunol 2019;208:631-640.

19. Herrera-Ramos E, Lopez-Rodriguez M, Ruiz-Hernandez JJ, Horcajada JP, Borderias L, et al. Surfactant protein A genetic variants associate with severe respiratory insufficiency in pandemic influenza A virus infection. Crit Care 2014;18:R127.

20. Cheng Z, Zhou J, To KK-W, Chu H, LiC, et al. Identification of TMPRSS2 as a susceptibility gene for severe 2009 pandemic A(H1N1) influenza and A(H7N9) influenza. J Infect Dis 2015;212:1214-1221.

21. Sologuren I, Martinez-Saavedra MT, Sole-Violan J, de Borges de Oliveira E, Betancor $\mathrm{E}$, et al. Lethal influenza in two related adults with inherited GATA2 deficiency. J Clin Immunol 2018;38:513-526.

22. Bravo García-Morato M, Calvo Apalategi A, Bravo-Gallego LY, Blázquez Moreno A, Simón-Fuentes M, et al. Impaired control of multiple viral infections in a family with complete IRF9 deficiency. J Allergy Clin Immunol 2019;144:309-312.

23. Ciancanelli MJ, Huang $S$, Luthra $P$, Garner $H$, Itan $Y$, et al. Infectious disease. Life-threatening influenza and impaired interferon amplification in human IRF7 deficiency. Science 2015;348:448-453

24. Hernandez N, Melki I, Jing H, Habib T, Huang SSY, et al. Lifethreatening influenza pneumonitis in a child with inherited IRF9 deficiency. J Exp Med 2018;215:2567-2585.

25. Esposito S, Molteni CG, Giliani S, Mazza C, Scala A, et al. Tolllike receptor 3 gene polymorphisms and severity of pandemic $\mathrm{A} / \mathrm{H} 1 \mathrm{~N} 1 / 2009$ influenza in otherwise healthy children. Virol J 2012:9:270.

26. Hidaka F, Matsuo S, Muta T, Takeshige K, Mizukami T, et al. A missense mutation of the Toll-like receptor 3 gene in a patient with influenza-associated encephalopathy. Clin Immunol 2006:119:188-194.

27. Lim HK, Huang SXL, Chen J, Kerner G, Gilliaux O, et al. Severe influenza pneumonitis in children with inherited TLR3 deficiency. J Exp Med 2019;216:2038-2056.

28. Chen Y, Zhou J, Cheng Z, Yang S, Chu H, et al. Functional variants regulating LGALS1 (Galectin 1) expression affect human susceptibility to influenza A(H7N9. Sci Rep 2015;5:8517.

29. Nogales A, L DeDiego M. Host single nucleotide polymorphisms modulating influenza A virus disease in humans. Pathogens 2019;8.

30. Fodor E, Te Velthuis AJW. Structure and function of the influenza virus transcription and replication machinery. Cold Spring Harb Perspect Med 2019;10.

31. Wandzik JM, Kouba T, Cusack S. Structure and function of influenza polymerase. Cold Spring Harb Perspect Med 2020:a038372.

32. Staller E, Barclay WS. Host cell factors that interact with influenza virus Ribonucleoproteins. Cold Spring Harb Perspect Med 2020.

33. Carrique L, Fan H, Walker AP, Keown JR, Sharps J, et al. Host ANP32A mediates the assembly of the influenza virus replicase. Nature 2020;587:638-643.

34. Chang S, Sun D, Liang H, Wang J, Li J, et al. Cryo-EM structure of influenza virus RNA polymerase complex at $4.3 \AA$ resolution. Mol Cell 2015:57:925-935.

35. Chen KY, Santos Afonso ED, Enouf V, Isel C, Naffakh N. Influenza virus polymerase subunits co-evolve to ensure proper levels of dimerization of the heterotrimer. PLoS Pathog 2019;15:e1008034.

36. Fan H, Walker AP, Carrique L, Keown JR, Martin S, et al. Structures of influenza A virus RNA polymerase offer insight into viral genome replication. Nature 2019;573:287-290.

37. Peng $Q$, Liu $Y$, Peng $R$, Wang $M$, Yang $W$, et al. Structural insight into RNA synthesis by influenza D polymerase. Nat Microbiol 2019:4:1750-1759.

38. Long JS, Giotis ES, Moncorge O, Frise R, Mistry B, et al. Species difference in ANP32A underlies influenza A virus polymerase host restriction. Nature 2016;529:101-104.

39. Staller E, Sheppard CM, Neasham PJ, Mistry B, Peacock TP, et al. ANP32 proteins are essential for influenza virus replication in human cells. J Virol 2019;93:17. 
40. Zhang $H$, Zhang Z, Wang $Y$, Wang $M$, Wang $X$, et al. Fundamental contribution and host range determination of ANP32A and ANP32B in influenza A virus polymerase activity. J Virol 2019;93:13.

41. Lonsdale J, Thomas J, Salvatore M, Phillips R, Lo E, et al. The GenotypeTissue Expression (GTEx) project. Nat Genet 2013;45:580-585.

42. Reilly PT, Yu Y, Hamiche A, Wang L. Cracking the ANP32 whips: important functions, unequal requirement, and hints at disease implications. Bioessays 2014;36:1062-1071.

43. Kleiner RE, Hang LE, Molloy KR, Chait BT, Kapoor TM. A chemical proteomics approach to reveal direct protein-protein interactions in living cells. Cell Chem Biol 2018;25:110-120.

44. Obri A, Ouararhni K, Papin C, Diebold ML, Padmanabhan K, et al. ANP32E is a histone chaperone that removes H2A.Z from chromatin. Nature 2014:505:648-653

45. Saavedra F, Rivera C, Rivas E, Merino P, Garrido D, et al. PP32 and SET/TAF- $\mid \beta$ proteins regulate the acetylation of newly synthesized histone H4. Nucleic Acids Res 2017:45:11700-11710.

46. Tochio N, Umehara T, Munemasa Y, Suzuki T, Sato S, et al. Solution structure of histone chaperone ANP32B: interaction with core histones $\mathrm{H} 3-\mathrm{H} 4$ through its acidic concave domain. J Mol Biol 2010;401:97-114.

47. Camacho-Zarco AR, Kalayil S, Maurin D, Salvi N, Delaforge E, et al. Molecular basis of host-adaptation interactions between influenza virus polymerase PB2 subunit and ANP32A. Nat Commun 2020;11:3656.

48. Huyton $T$, Wolberger $C$. The crystal structure of the tumor suppressor protein pp32 (Anp32a): structural insights into Anp32 family of proteins. Protein Sci 2007;16:1308-1315.

49. Long JS, Idoko-Akoh A, Mistry B, Goldhill D, Staller E, et al. Species specific differences in use of ANP32 proteins by influenza A virus. eLife 2019;8:e45066.

50. Peacock TP, Sheppard CM, Staller E, Frise R, Swann OC, et al. Mammalian ANP32A and ANP32B proteins drive alternative avian influenza virus polymerase adaptations. bioRxiv 2020;2020.09.03.282384

51. Mistry B, Long JS, Schreyer J, Staller E, Sanchez-David RY, et al. Elucidating the interactions between influenza virus polymerase and host factor ANP32A. J Virol 2020;94:e01353-01319.
52. Kaltenegger E, Ober D. Paralogue interference affects the dynamics after gene duplication. Trends Plant Sci 2015;20:814-821.

53. Karczewski KJ, Francioli LC, Tiao G, Cummings BB, Alföldi J, et al. Variation across 141,456 human exomes and genomes reveals the spectrum of loss-of-function intolerance across human proteincoding genes. bioRxiv 2019;531210.

54. Hong R, Macfarlan T, Kutney SN, Seo S-B, Mukai Y, et al. The identification of phosphorylation sites of PP32 and biochemical purification of a cellular PP32-kinase. Biochemistry 2004:43:10157-10165

55. Taliun D, Harris DN, Kessler MD, Carlson J, Szpiech ZA, et al. Sequencing of 53,831 diverse genomes from the NHLBI TOPMed Program. bioRxiv 2019;563866.

56. Peacock TP, Swann OC, Staller E, Leung PB, Goldhill DH, et al. Swine ANP32A supports avian influenza virus polymerase. bioRxiv 2020;2020.01.24.916916

57. Beck S, Zickler M, Pinho Dos Reis V, Günther T, Grundhoff A et al. ANP32B deficiency protects mice from lethal influenza a virus challenge by dampening the host immune response. Front Immunol 2020;11:450.

58. Liang L, Jiang L, Li J, Zhao Q, Wang J, et al. Low polymerase activity attributed to PA drives the acquisition of the PB2 E627K mutation of H7N9 avian influenza virus in mammals. mBio 2019:10

59. Fries B, Heukeshoven J, Hauber I, Gruttner C, Stocking C, et al. Analysis of nucleocytoplasmic trafficking of the HuR ligand APRIL and its influence on CD83 expression. J Biol Chem 2007;282:4504-4515.

60. Giard DJ, Aaronson SA, Todaro GJ, Arnstein P, Kersey JH, et al. In vitro cultivation of human tumors: establishment of cell lines derived from a series of solid tumors. J Natl Cancer Inst 1973;51:1417-1423.

61. Graham FL, Smiley J, Russell WC, Nairn R. Characteristics of a human cell line transformed by DNA from human adenovirus type 5. J Gen Virol 1977;36:59-74.

62. Lin YC, Boone M, Meuris L, Lemmens I, Van Roy N, et al. Genome dynamics of the human embryonic kidney 293 lineage in response to cell biology manipulations. Nat Commun 2014;5:4767.

\section{Five reasons to publish your next article with a Microbiology Society journal}

1. The Microbiology Society is a not-for-profit organization.

2. We offer fast and rigorous peer review - average time to first decision is 4-6 weeks.

3. Our journals have a global readership with subscriptions held in research institutions around the world.

4. $80 \%$ of our authors rate our submission process as 'excellent' or 'very good'.

5. Your article will be published on an interactive journal platform with advanced metrics.

Find out more and submit your article at microbiologyresearch.org. 